\title{
Simulations, Field Modeling, and Systematic Analyses for Muon g-2 and EDM
}

\author{
Eremey Valetov
}

Lancaster University, Michigan State University, and the Cockcroft Institute

\section{December 19, 2019}

\section{Lancaster}

\section{$\frac{\text { MICHIGAN STATE }}{U \text { I I E R S I T Y }}$}

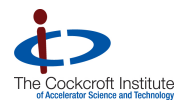

SJTU Special Seminar

This manuscript has been authored by Fermi Research Alliance, LLC under Contract No. DE-AC02-07CH11359 with the U.S. Department of Energy, Office of Science, Office of High Energy Physics. 
(1) Introduction

(2) Fringe Fields of Electrostatic Deflectors

(3) Main and Fringe Fields of the Muon $g$-2 Collaboration Quadrupole

(4) Investigation of Spin Decoherence and Systematic Errors in Frozen Spin and Quasi-Frozen Spin Lattices

(5) End-to-End Beamline Simulations for the Muon $g$-2 Experiment and Systematic Analyses 
Section 1

\section{Introduction}




\section{Particle Accelerators and Storage Rings}

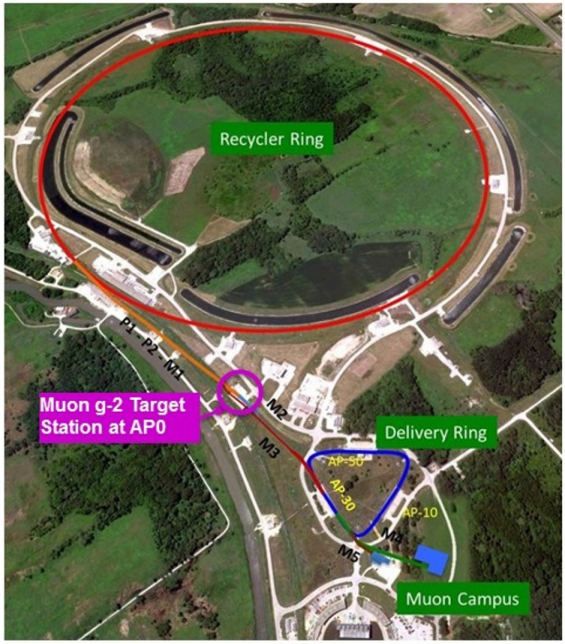

Recycler Ring and the Muon Campus at Fermilab 


\section{Magnetic Dipole Moment (MDM)}

- The magnetic dipole moment (MDM) $\mu$ is defined by the relation $\tau=\mu \times B$, where $\tau$ is the torque exerted on an object, such as a magnet, by an external magnetic field $B$.

- The spin MDM of a lepton (an electron $e^{-}$, a muon $\mu^{-}$, or a tau $\left.\tau^{-}\right)$is $\mu=g \frac{e}{2 m} s$, where the lepton spin is $s=1 / 2, m$ is the lepton mass, $e$ is the elementary charge, and $g$ is the $g$-factor (gyromagnetic ratio) of the lepton.

- The Dirac equation predicts the $g$ factor as 2 for leptons, and the quan-

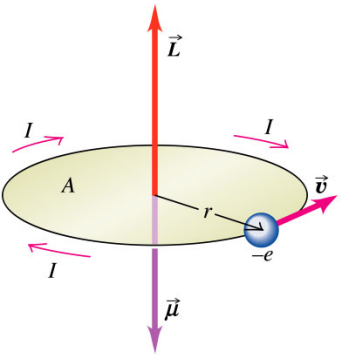

In the classical model, the orbital MDM of an electron arises from the electron orbiting the nucleus. (Image source: tity $a=(g-2) / 2$, arising from quantum effects, is known as anomalous MDM (or MDM anomaly). 


\section{Anomalous MDM Measurement}

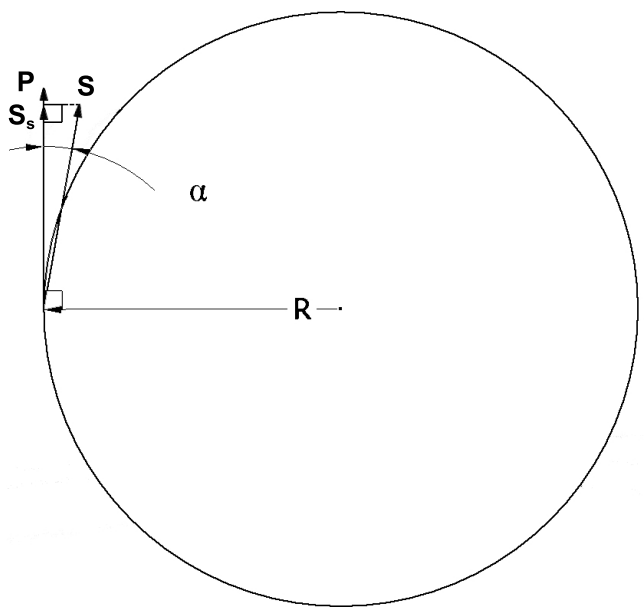

The Muon g-2 Experiment at Fermilab measures anomalous MDM using muons at the "magic" momentum $3.094 \mathrm{GeV} / \mathrm{c}$, where spin precession is proportional to the anomalous MDM. 


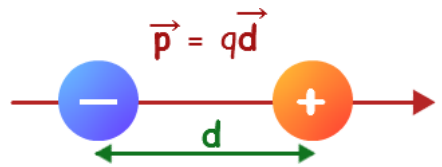

An electric dipole with EDM $p=q d$. (Image source: Wikipedia.)

- An electric dipole is a system characterized by centers of equal and opposite total charges $\pm q$ separated by a distance $d$.

- The electric dipole moment (EDM) of two point-like charges is defined as $p=q d$.

- EDMs of fundamental particles were not experimentally observed so far. 


\section{Implications for the Standard Model (SM) and Beyond-BSM Possibilities}

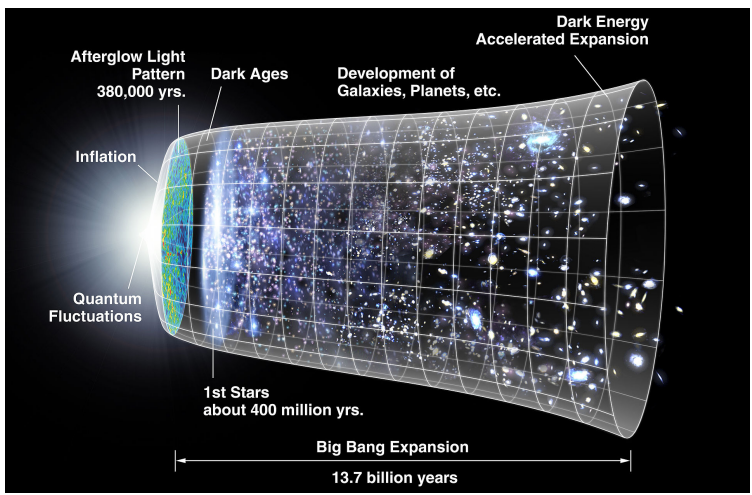

Evolution of the universe (image source: Wikipedia). 


\section{The Frozen Spin (FS) Method}

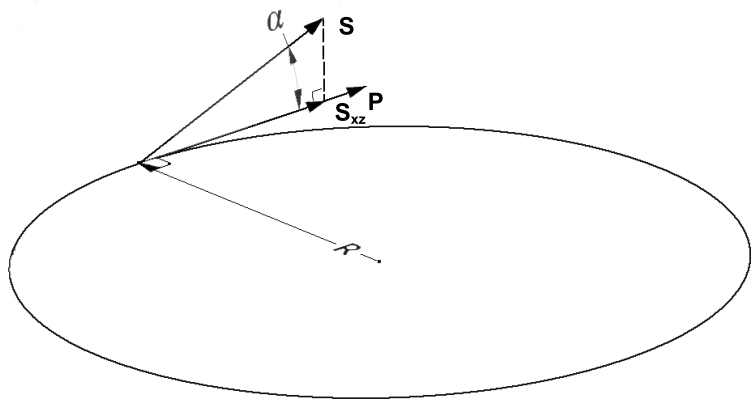

- In the frozen spin concept for the measurement of deuteron EDM, the spin and the momentum are horizontally aligned.

- An non-zero EDM would result in spin precession in the vertical plane. 


\section{Thomas-BMT Equation}

The Thomas-BMT equation describes the dynamics of spin vector $s$ in magnetic field $B$ and electrostatic field $E$, and it is generalized to account for the EDM effects as follows:

$$
\frac{d s}{d t}=s \times\left(\Omega_{\mathrm{MDM}}+\Omega_{\mathrm{EDM}}\right),
$$

where the MDM and EDM angular frequencies $\Omega_{\mathrm{MDM}}$ and $\Omega_{\mathrm{EDM}}$ are

$$
\begin{aligned}
& \Omega_{\mathrm{MDM}}=\frac{q}{m}\left[G B-\left(G-\frac{1}{\gamma^{2}-1}\right) \frac{E \times \beta}{c}\right], \\
& \Omega_{\mathrm{EDM}}=\frac{q}{m} \frac{\eta}{2}\left[\frac{E}{c}+\beta \times B\right],
\end{aligned}
$$

where $m, q, G$ are the particle mass, electric charge, and anomalous MDM, respectively; $\beta$ is the ratio of particle velocity to the speed of light; and $\gamma$ is the Lorentz factor. The EDM factor $\eta$ is defined by $d=\eta \frac{q}{2 m c} s$, where $d$ is the particle EDM and $s$ is the particle spin. 
Section 2

Fringe Fields of Electrostatic Deflectors 


\section{Conformal Mapping Methods}

- A conformal mapping (or conformal map) is a transformation $f: \mathbb{C} \rightarrow \mathbb{C}$ that is locally angle preserving.

- A conformal mapping satisfies Cauchy-Riemann equations and, therefore, its real and imaginary parts satisfy Laplace's equation: $\Delta \Re(f)=0$ and $\Delta \Im(f)=0$.

- Conformal mappings automatically provide the electrostatic potential in cases when the problem geometry can be represented by a polygon, possibly with some vertices at the infinity.

- The domain of a conformal mapping is called the canonical domain, and the image of a conformal mapping is called the physical domain.

- A Schwarz-Christoffel (SC) mapping is a conformal mapping from the upper half-plane as the canonical domain to the interior of a polygon as the physical domain. 


\section{Example of a Schwartz-Christoffel Mapping}

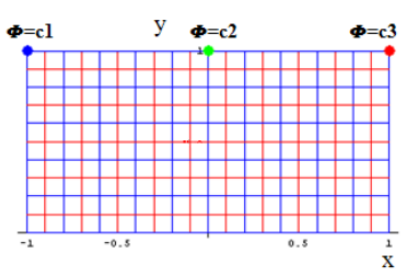

a. z plane

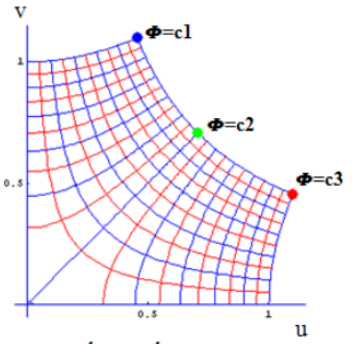

b. w plane

The Schwartz-Christoffel mapping $f(z)=\sqrt{z}$ maps the upper half-plane to the upper-right quadrant of the complex plane. (Image source: Kapania et al.) 


\section{Fringe Fields of Semi-Infinite Capacitors}

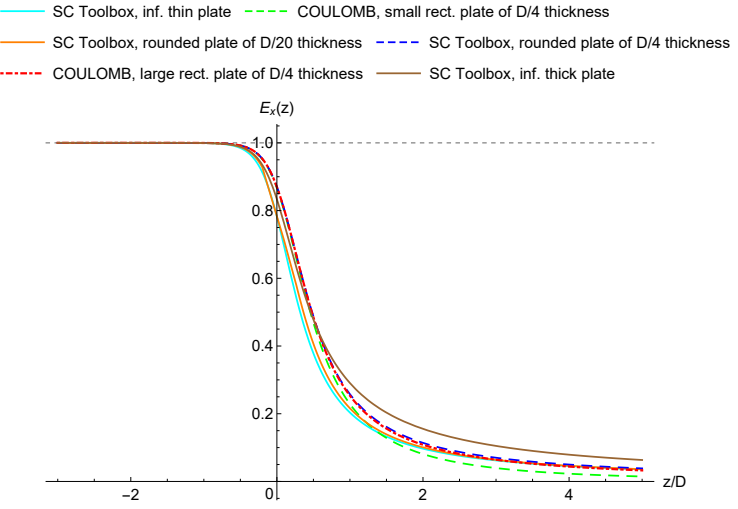

Comparison of field falloffs of several semi-infinite capacitors computed in the SC Toolbox with field falloffs of two finite rectangular capacitors computed in COULOMB. 


\section{Fringe Fields of Two Adjacent Semi-Infinite Capacitors}
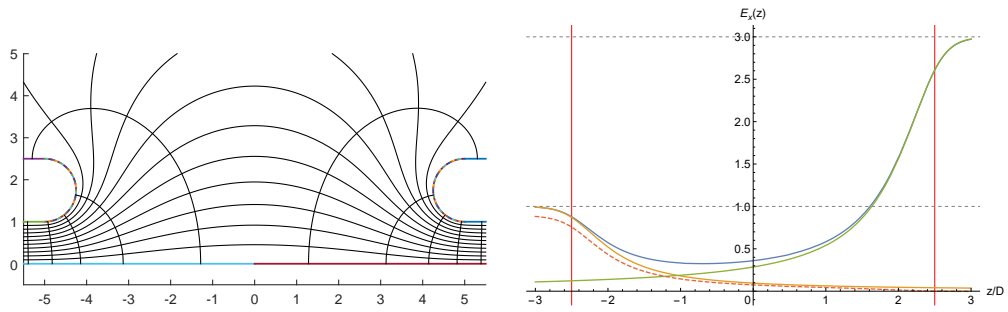

Additionally, we modeled fringe fields of two adjacent semi-infinite capacitors with finitely thick plates and symmetric, antisymmetric, and different voltages. 


\section{Accurate Fringe Fields Representation}

- We found that the field falloff of an electrostatic deflector is slower than exponential.

- Enge functions of the form $F_{N}(z)=\frac{1}{1+\exp \left(\sum_{j=\mathbf{1}}^{N} a_{j}\left(\frac{z}{D}\right)^{j-1}\right)}$ are not suitable for accurate modeling of such falloffs.

- We proposed an alternative function

$$
\begin{aligned}
H(z) & =\frac{1}{1+\exp \left[\sum_{j=1}^{N_{1}} a_{j}\left(\frac{z}{D}\right)^{j-1}\right]} \frac{1}{1+\exp \left[\left(\frac{z}{D}-c\right)^{2}\right]}+ \\
& +\frac{1}{\sum_{j=1}^{N_{2}} b_{j}\left(\frac{z}{D}\right)^{j-1}} \frac{1}{1+\exp \left[-\left(\frac{z}{D}-c\right)^{2}\right]}
\end{aligned}
$$

to model field falloffs of electrostatic deflectors. 


\section{Accurate Fringe Fields Representation}
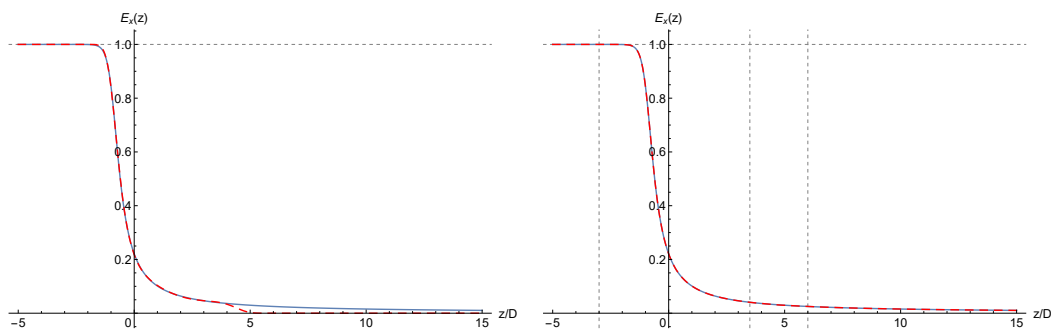

A function of the form $H(z)$ provides a good approximation of the fringe field of an electrostatic deflector (right), in contrast to an Enge function (left). 
Section 3

\section{Main and Fringe Fields of the Muon $g-2$ Collaboration Quadrupole}




\section{Main Field of the Muon g-2 Collaboration Quadrupole}

The main field of the Muon g-2 collaboration quadrupole may be obtained using the following general method:

(1) Calculate the electrostatic potential using conformal mapping methods with one plate - the left plate on the cross section drawing - at $1 \mathrm{~V}$ and the other Dirichlet boundary conditions (the remaining plates, the rectangular enclosure, and the trolley rails) of $0 \mathrm{~V}$.

(2) Apply plate distance errors as perturbations to four copies of the potential, each copy corresponding to one plate at $1 \mathrm{~V}$ and the other Dirichlet boundary conditions of $0 \mathrm{~V}$.

(3) Apply appropriate rotations to these four copies of the potential, scale the copies (e.g., by $\pm 2.4 \times 10^{4}$ or with mispowered values), and use their superposition.

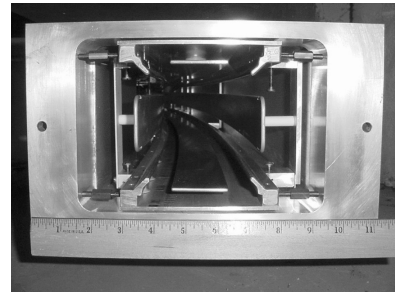

The Muon g-2 collaboration quadrupole.

(Image source: Semertzidis et al.)

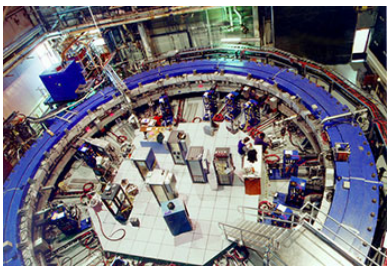

The Muon g-2 ring at Fermilab. (Image source: FNAL.) 


\section{Nominal Symmetric and Non-Symmetric Models}
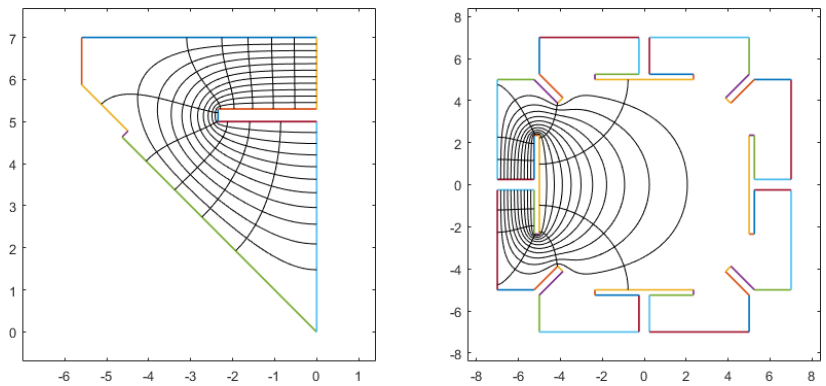

The plots on the left and right show the polygonal model of the Muon $g$-2 collaboration quadrupole in the symmetric (SM) and non-symmetric (NSM) cases, respectively. 


\section{Conformal Mapping Derivative}

In both cases, the derivative of the conformal map $f$ from the canonical domain to the physical domain is

$$
f^{\prime}(z)=c \operatorname{cn}(z \mid m) \operatorname{dn}(z \mid m) \prod_{j=1}^{n}\left(\operatorname{sn}(z \mid m)-\operatorname{sn}\left(x_{j}+i y_{j} \mid m\right)\right)^{\alpha_{j}-1},
$$

where sn, cn, and $\mathrm{dn}$ are the Jacobi elliptic functions ${ }^{1}, \mathrm{~K}$ is the complete elliptic integral of the first kind ${ }^{2}$, the parameters $n$ and $\alpha$ were obtained from the polygonal model, and the parameters $x, y, m$, and $c$ were found using the SC Toolbox.

${ }^{1}$ Definitions of the Jacobi elliptic functions can be found at http://mathworld.wolfram.com/JacobiEllipticFunctions.html.

${ }^{2}$ The complete elliptic integral of the first kind is defined at http://mathworld.wolfram.com/

CompleteEllipticIntegralof theFirstKind.html. 


\section{Multipole Terms}
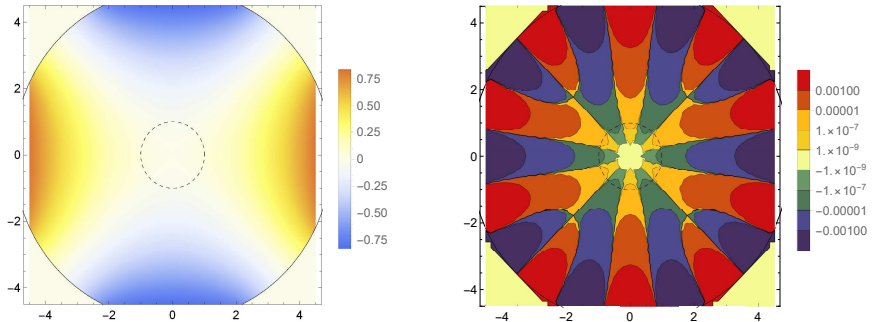

- We obtained the multipole expansion of the electrostatic potential in both SM and NSM cases to order 24 using the differential algebra (DA) inverse of the conformal mapping, as well as using Fourier analysis.

- The use of conformal mapping methods for the calculation of the main field has the advantage of an analytic, fully Maxwellian formula and allows rapid recalculations with adjustments to the geometry and mispowered plates. 


\section{Fringe Field of the Muon $g$-2 Collaboration Quadrupole}

- We obtained the quadrupole strength falloff and the EFB $z_{\mathrm{EFB}}=1.2195 \mathrm{~cm}$ for the Muon $g-2$ collaboration quadrupole by calculating Fourier modes of its electrostatic potential at a set of radii in the transversal plane.

- The respective electrostatic potential data was obtained using COULOMB's BEM field solver from a $3 \mathrm{D}$ model of the quadrupole.

- For a confirmatory comparison, we applied the same method of calculating multipole strengths to the electrostatic field data obtained for the Muon $g-2$ collaboration quadrupole using Opera-3d's finite element method (FEM) field solver by Wanwei Wu (FNAL).

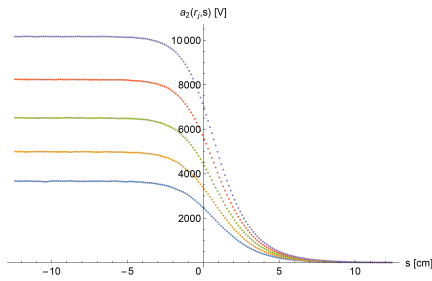

Falloffs of 2nd order Fourier modes $a_{2}\left(r_{j}\right)$ calculated at radii $r=1.8,2.1,2.4,2.7,3.0 \mathrm{~cm}$ from Wu's field data. Curves with larger magnitudes correspond to larger radii. 


\section{Results Based on Soltner-Valetov and Wu Field Data}

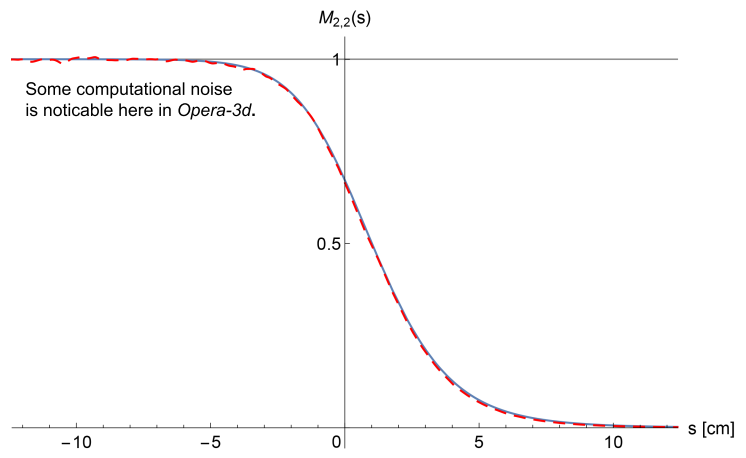

The field falloffs and the EFBs obtained from Soltner-Valetov and Wu field data are in good agreement, and they explained the experimentally measured Muon $g$-2 ring tunes. 


\section{Section 4}

\section{Investigation of Spin Decoherence and Systematic Errors in Frozen Spin and Quasi-Frozen Spin Lattices}




\section{Quasi-Frozen Spin (QFS) Concept}

- The quasi-frozen spin (QFS) lattice concept is based on the FS concept, but the requirement that spin needs to be aligned with momentum is relaxed: in QFS, spin is aligned with momentum on average during each turn.

- The QFS condition to maintain an average alignment of spin with momentum is $\theta_{B}+\theta_{E}=0$, where $\theta_{B}$ and $\theta_{E}$ are the polar rotation angles of spin relative to momentum in the magnetic field and electrostatic field, respectively.

- This yields the QFS condition in terms of momentum rotation as

$$
\gamma G \Phi_{B}+\beta^{2} \gamma\left(\frac{1}{\gamma^{2}-1}-G\right) \Phi_{E}=0 .
$$




\section{Lattice parameters}

Length: $166.67 \mathrm{~m}$

Particles: deuterons

Kinetic energy: $270 \mathrm{MeV}$

- 4 straight sections (light gray)

- 4 magnetic sections (blue)

- 4 electrostatic sections (green)

\section{System plot}

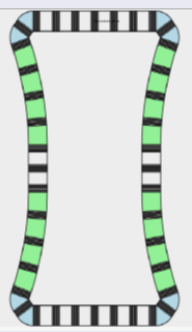

\section{Decoherence order suppression}

- RF cavity: 1st and, partially, 2nd order (by mixing the particles relatively to the average field strength, averaging out $\triangle \gamma G$ for each particle).

- Sextupoles: remaining 2nd order component, (which is due to the average of $\triangle \gamma G$ being different for each particle). 


\section{Lattice parameters}

Length: $149.21 \mathrm{~m}$

Particles: deuterons

Kinetic energy: $270 \mathrm{MeV}$

- 2 straight sections (light gray)

- 4 magnetic sections (blue)

- $2 \mathrm{E}+\mathrm{B}$ sections (orange)

\section{System plot}

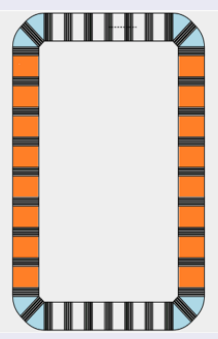

\section{Decoherence order suppression}

- RF cavity: 1st and, partially, 2nd order

- Sextupoles: remaining 2nd order component

The E+B static Wien Filter elements are used instead of the electrostatic deflector (1) to remove nonlinear components due to curved electrostatic element and (2) to simplify the system from the engineering perspective. 


\section{Lattice parameters}

Length: $145.85 \mathrm{~m}$

Particles: deuterons

Kinetic energy: $270 \mathrm{MeV}$

- 2 straight sections (light gray)

- 2 curved E+B sections (light blue)

\section{System plot}

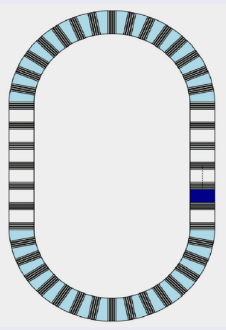

\section{Decoherence order suppression}

- RF cavity: 1 st and partially 2 nd order

- Sextupoles: remaining 2nd order component

The design of this lattice is based on the FS method and uses a curved $E+B$ element as proposed by the Storage Ring Electric Dipole Moment Collaboration. 


\section{Optimization of Sextupole Strengths}

- 'RF'x'SFP1'S6p3FR344p4MHz100kVa' - 'RF'x'SDP1'S6p3FR344p4MHz100kVa'

- 'RF'x SDP2 S6p3FR3 4p4MHz100kVa' . RF' x SFN1'S6p3FR3f4p4MHz100kVa'

- 'RF'x'SDN1'S6p3FR3f4p4MHz100kVa' - 'RF'x'SDN2'S6p3FR3 4 p4MHz100kVa'

- 'RF'x'SFP1'S6p3FR344p4MHz100kVb' - RF' x'SDP1'S6p3FR344p4MHz100kVb'

- 'RF' ' 'SDP2 S6p3FR3f4p4MHz100kVb' . RF'x'SFN1'S6p3FR3f4p4MHz100kVb'

- 'RF'x SDN1'S6p3FR344p4MHz100kVb' 'RF'x'SDN2 S6p3FR3f4p4MHz100kVb

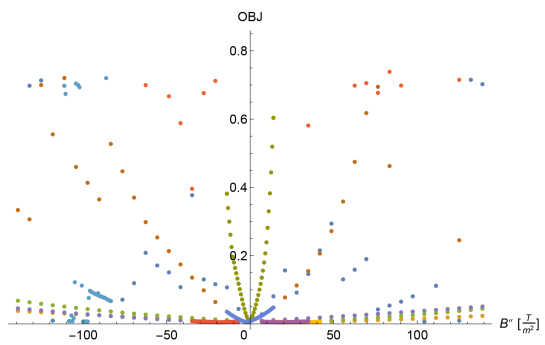

Objective function $\mathrm{OBJ}$ as a function of sextupole family strengths in the Senichev 6.3 QFS lattice. Tracking was performed with the RF cavity on, particles launched with horizontal offsets up to $x_{i}= \pm 5 \times 10^{-3} \mathrm{~m}$, and fringe field mode FR 3 . 


\section{Spin Decoherence with Optimized Sextupole Strengths}

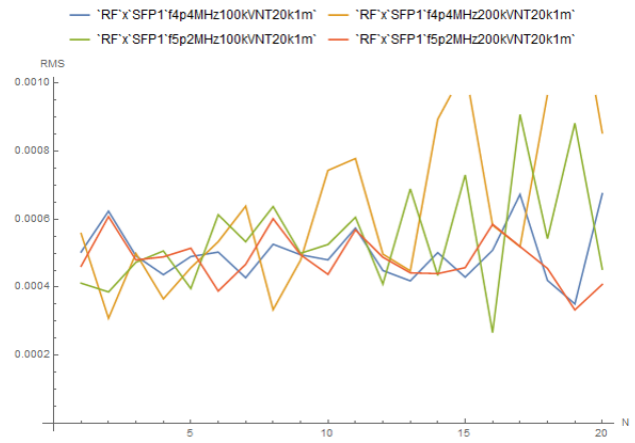

- With an optimized sextupole family strength, the spin decoherence often remains in the same range for at least $4.2 \times 10^{5}$ turns.

- The QFS structure decoherence is qualitatively and quantitatively similar to that of a FS structure decoherence. 


\section{Systematic Errors due to Magnet Rotational Misalignments}
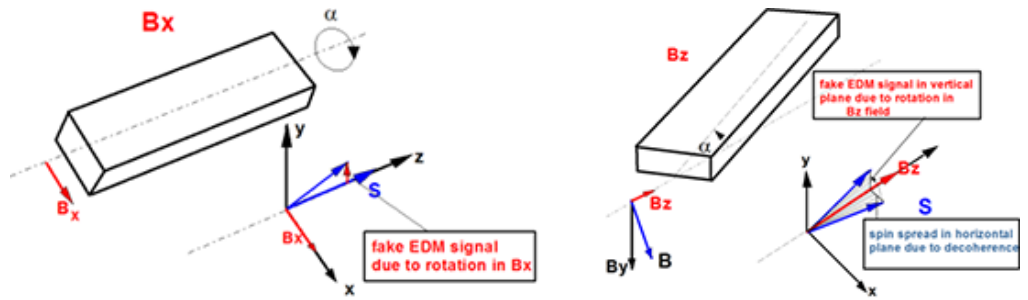

The rotational magnet misalignments, $B_{x}$ and $B_{z}$ error field components.

We studied the effect of rotational magnet misalignments on spin dynamics, namely spin decoherence and frequencies of rotation in a vertical plane, in QFS and FS structures. The error field components $B_{x}$ and $B_{z}$ are the most relevant to the detection of an EDM signal. 


\section{Mitigation of $B_{x}$ and $B_{z}$ Error Components}

\section{Clockwise (CW) and counterclockwise (CCW) lattice traversal}

- We proposed to track polarized particle bunches in the QFS/FS lattices in both $\mathrm{CW}$ and $\mathrm{CCW}$ directions.

- We consider the $\mathrm{CW}$ direction to be forward and the CCW direction to be reverse.

- We use the fact that in the linear approximation the reverse spin transfer map coincides with the inverse spin transfer map.

\section{$B_{x}$ error field component}

- Rotation frequencies are $\Omega_{x}^{\mathrm{CW}}=\Omega_{B_{x}}^{\mathrm{CW}}+\Omega_{\mathrm{EDM}}$ and $\Omega_{x}^{\mathrm{CCW}}=-\Omega_{\mathrm{B}_{x}}^{\mathrm{CCW}}+\Omega_{\mathrm{EDM}}$ in the vertical plane and $\Omega_{y}=0+\left\langle\delta \Omega_{\mathrm{decoh}}\right\rangle$ in the horizontal plane.

- It is necessary to (1) minimize the decoherence in the vertical plane $\sigma\left(\Omega_{B_{x}}\right)$ the same way as in the horizontal plane using the RF cavity and sextupole families and (2) minimize $\left|\Omega_{B_{x}}^{\mathrm{CW}}-\Omega_{B_{x}}^{\mathrm{CCW}}\right|$.

- Rotation frequency due to EDM is obtained by $\Omega_{\mathrm{EDM}}=\left(\Omega_{x}^{\mathrm{CW}}+\Omega_{x}^{\mathrm{CCW}}\right) / 2+\left(\Omega_{B_{x}}^{\mathrm{CCW}}-\Omega_{B_{x}}^{\mathrm{CW}}\right) / 2$. 


\section{Mitigation of $B_{X}$ and $B_{z}$ Error Components}

\section{$B_{z}$ error field component}

- The method of error field component mitigation for $B_{x}$ is not applicable to $B_{z}$.

- We have to minimize $\Omega_{B_{z}}$ to $\sim 10^{-10} \frac{\mathrm{rad}}{\mathrm{s}}$ using additional trim coils.

\section{Outcome of the $B_{x}$ and $B_{z}$ error component mitigation method}

- For the error component mitigation method outlined here, we obtained a measurement accuracy estimate of $\Omega_{\mathrm{EDM}}$ is $\sim 10^{-9} \frac{\mathrm{rad}}{\mathrm{s}}$.

- As a result, the accuracy of EDM signal measurement in one run is $\sim 10^{-28} \mathrm{e} \cdot \mathrm{cm}$.

- The accuracy of the EDM signal measurement after one year of measurement may be $\sim 10^{-30} \mathrm{e} \cdot \mathrm{cm}$. 


\section{Vertical Spin Decoherence, Exact QFS/FS}

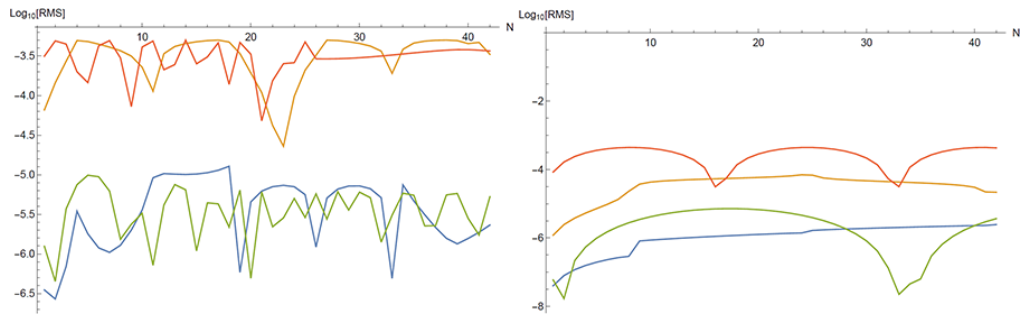

Our systematic errors study shows that, for at least $4.2 \times 10^{5}$ turns, the vertical spin decoherence due to rotational magnet misalignments often remained in the same range (or grew within the range of the spin decoherence curve for tracking in the opposite direction) in both FS (Senichev BNL) and QFS (Senichev E+B) lattices. 


\section{Section 5}

\section{End-to-End Beamline Simulations for the Muon g-2 Experiment and Systematic Analyses}




\section{End-to-End Beamline Simulations}

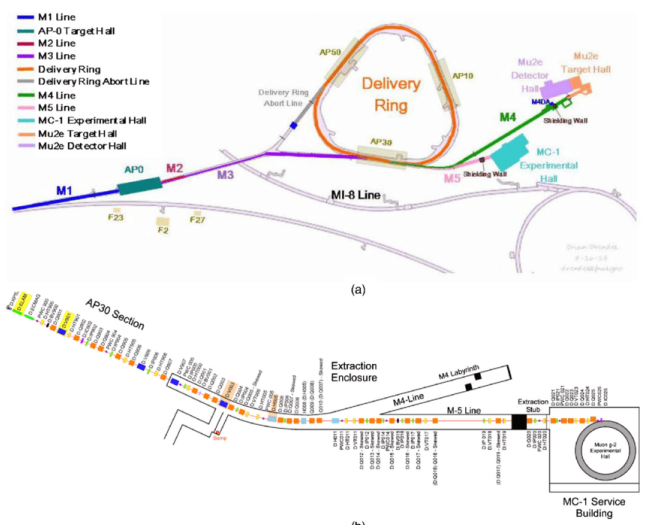

(b)

Using high-performance computing resources and simulation codes G4Beamline and BMAD, we performed end-to-end beamline simulations for the Muon g-2 Experiment with $3 \times 10^{12}$ protons-on-target. 


\section{MARS Model of the Target Station}

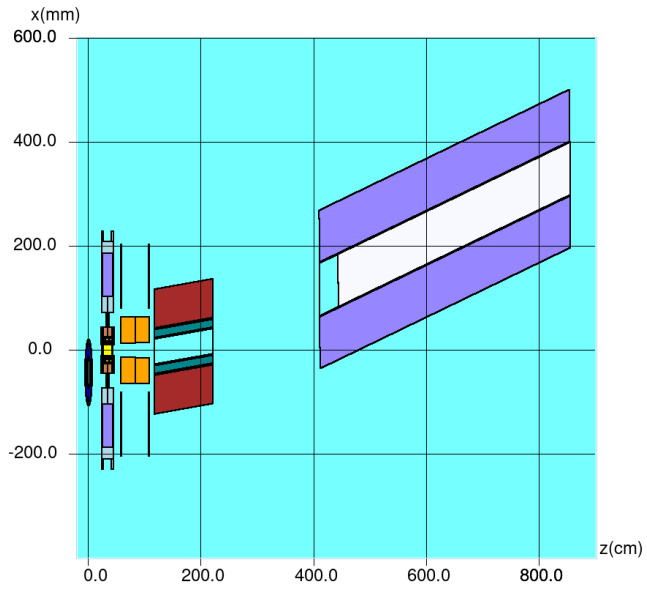

We revised the MARS model of the Muon $g$-2 target station AP0 from its 2011 version. MARS is a Monte Carlo code often used for target station and detector simulations. 


\section{Muon Losses Study}
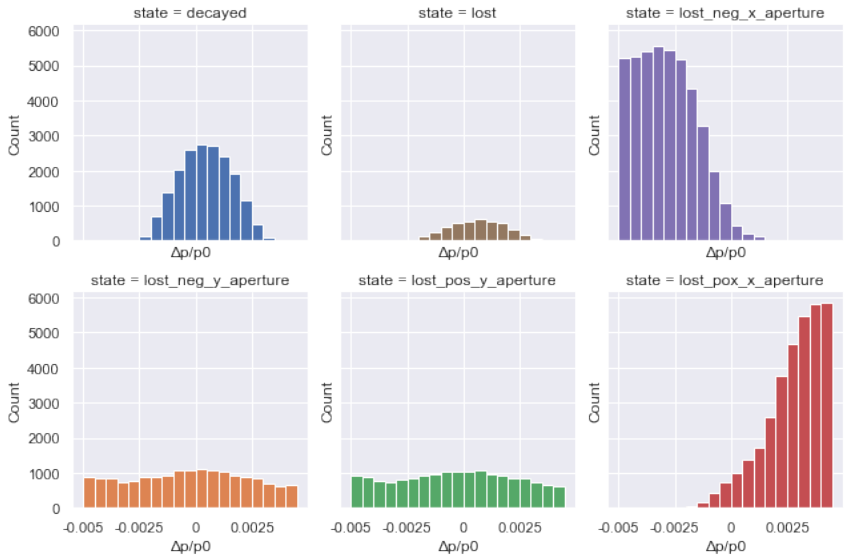

We continued the end-to-end beamline simulations for 2000 turns around the Muon $g$-2 storage ring, and studied the momentum-dependent muon losses, which cause a systematic shift of the measured anomalous MDM. 


\section{Acknowledgments}

- This material is based upon work supported by the U.S. Department of Energy, Office of Science, under Contract No. DE-FG02-08ER41546 and Contract No. DE-SC0018636.

- This research was supported by the Cockcroft Institute of Accelerator Science and Technology, a Science and Technology Facilities Council facility.

- This document was prepared by the Muon $g-2$ collaboration using the resources of the Fermi National Accelerator Laboratory (Fermilab), a U.S. Department of Energy, Office of Science, HEP User Facility. Fermilab is managed by Fermi Research Alliance, LLC (FRA), acting under Contract No. DE-AC02-07CH11359.

- This research used resources of the National Energy Research Scientific Computing Center (NERSC), a U.S. Department of Energy Office of Science User Facility operated under Contract No. DE-AC02-05CH11231.

- This research was done using resources provided by the Open Science Grid, which is supported by the National Science Foundation award 1148698, and the U.S. Department of Energy's Office of Science. 


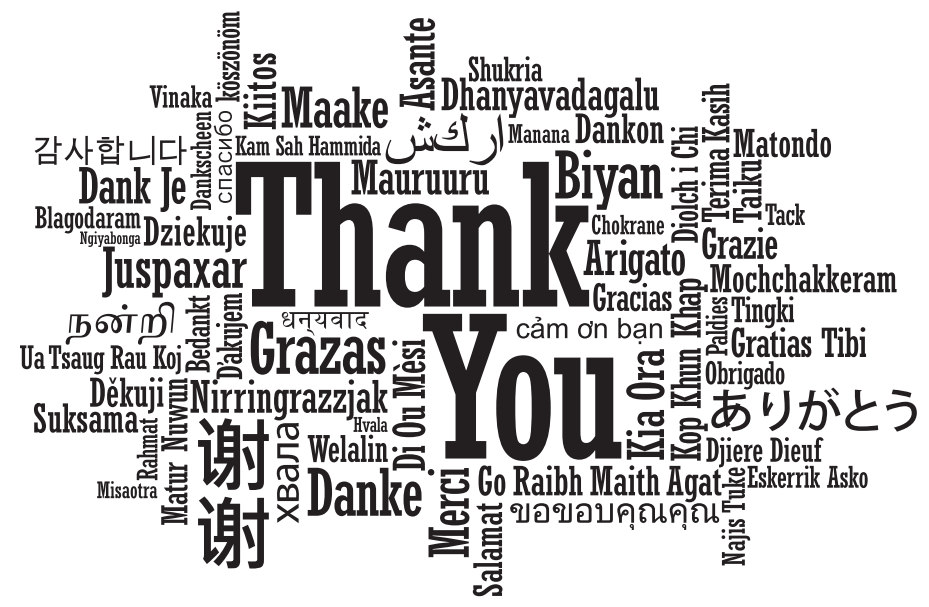

